\title{
ON HEARING THE SHAPE OF A TRIANGLE
}

\author{
PEI-KUN CHANG AND DENNIS DETURCK
}

(Communicated by Jonathan M. Rosenberg)

\begin{abstract}
To determine whether two triangles in the Euclidean plane are congruent, it suffices to know that they have their first $N$ eigenvalues in common, where $N$ depends on the first two eigenvalues of the triangles. Similar results for other figures are given.
\end{abstract}

\section{INTRODUCTION}

When discussing geometric properties of Riemannian manifolds which can be determined from the spectrum of the Laplacian operator of the manifolds, it has become fashionable to say that such a property can be "heard" (following $\mathrm{M}$. Kac $[\mathrm{K}])$. However, this kind of hearing is very idealized, in that most of the geometric information is obtained from the asymptotics of the sequence $\left\{\lambda_{n}\right\}$ of eigenvalues as $n$ tends to infinity. On the other hand, it is realistically possible to hear only a finite part of the spectrum, since the energies of the highfrequency components of vibrations must be very small in order for the total energy of the vibrations to be finite. The type of results that concern us here, therefore, address what one can discern from a finite part of the spectrum of the Laplacian. In particular, we consider the problem of differentiating among the members of a finite-dimensional family of metrics on a given manifold by means of a finite part of the Laplace spectrum. The first result of this sort was proved by $P$. Buser and $G$. Courtois for constant-curvature metrics on Riemann surfaces. In $\S 1$, a general theorem to this effect based on that of Buser and Courtois is proved, and is applied in $\S 2$ to the case of triangles in the Euclidean plane, and other figures in Euclidean space. These results provide a partial answer to a question posed by $M$. Potter in [P]. The key ingredients in the proof are the assumption of real-analytic dependence of the metric on the parameters, and the resulting real-analytic dependence of certain symmetric functions of the eigenvalues, and finally the fact that the ring of germs of realanalytic functions of finitely many variables is Noetherian. As pointed out in

Received by the editors June 9, 1988 and, in revised form, August 1, 1988.

1980 Mathematics Subject Classification (1985 Revision). Primary 58G25; Secondary 35P15.

Key words and phrases. Eigenvalues of Laplacian, inverse spectral problem.

The first author was supported by NATO Subvention 0153/87.

The second author was supported by NSF Grant MCS 85-03302 and NATO Subvention 0153/87. 
$\S 2$, extra geometric information about the family in question can help improve the conclusion of the result.

\section{Finitely many eigenvalues SUfFice}

Let $D$ be a compact oriented manifold (with or without boundary) of dimension $n$. We consider a family of metrics $g(\varepsilon)$, depending analytically on the parameter $\varepsilon \in \mathbb{R}^{p}$. Let $\lambda_{k}(\varepsilon)$ denote the $k$ th eigenvalue of the Laplacian of $g(\varepsilon)$ on $D$-if $\partial D \neq \varnothing$ we assume that $\partial D$ is piecewise smooth and we impose Dirichlet boundary conditions on the eigenfunctions. We also let $\operatorname{Spec}(\varepsilon)$ denote the entire collection of eigenvalues (counting multiplicities) of the Laplacian of $g(\varepsilon)$.

Proposition. With the notation above, for each compact subset $K$ of the parameter space, there is an integer $N(K)$ with the following property: If $\varepsilon_{0}, \varepsilon_{1} \in K$ such that $\lambda_{1}\left(\varepsilon_{0}\right)=\lambda_{1}\left(\varepsilon_{1}\right), \lambda_{2}\left(\varepsilon_{0}\right)=\lambda_{2}\left(\varepsilon_{1}\right), \ldots, \lambda_{N}\left(\varepsilon_{0}\right)=\lambda_{N}\left(\varepsilon_{1}\right)$, then $\operatorname{Spec}\left(\varepsilon_{0}\right)=$ $\operatorname{Spec}\left(\varepsilon_{1}\right)$. In other words, for $\varepsilon \in K$ the entire spectrum of the Laplacian of $g(\varepsilon)$ is determined by the values of the first $N(K)$ eigenvalues.

Proof. Since the metric depends analytically on the parameters, we can mimic the proofs of results of Rellich ([R], p. 83ff) and of Garabedian and Schiffer ([GS], Chapter V) on "interior variations" of domains to show that the Green's operator (resolvent) of the Laplacian of $g(\varepsilon)$ also varies analytically with $\varepsilon$. The Green's operator is compact (use the Green's operator for $\Delta+1$ in the case of manifolds without boundary), so we can use theorems from [Ko] as follows: Fix $\varepsilon_{0}$ in the parameter space and consider $g\left(\varepsilon_{0}\right)$. The multiplicities of the eigenvalues of the Laplacian of $g$ being finite, we can construct an increasing sequence $\left\{a_{i}\right\}$ with $a_{i} \uparrow \infty$ such that $a_{i} \notin \operatorname{Spec}\left(\varepsilon_{0}\right)$ and the number of linearly independent eigenfunctions with eigenvalue between $a_{i}$ and $a_{i+1}$ is finite for each $i$. By Theorem VII.1.7 of [Ko], the projection $P_{i}(\varepsilon)$ onto the finite-dimensional space spanned by the eigenfunctions of the Laplacian of $g(\varepsilon)$ with eigenvalue between $a_{i}$ and $a_{i+1}$ is analytic in $\varepsilon$ for $\varepsilon$ in some neighborhood of $\varepsilon_{0}$. Since the Laplacian of $g(\varepsilon)$ commutes with $P(\varepsilon)$, the nonzero eigenvalues of the composition of the two are the same as the eigenvalues of the Laplacian of $g(\varepsilon)$ between $a_{i}$ and $a_{i+1}$. Thus, we may consider the "piece" of the Laplacian between $a_{i}$ and $a_{i+1}$ to be the restriction of this composition to the image of $P_{i}(\varepsilon)$. The symmetric functions of the eigenvalues of this finite-dimensional operator completely determine and are determined by the eigenvalues themselves, and the symmetric functions of the eigenvalues are also analytic in $\varepsilon$.

Now we can begin the proof of the theorem in earnest. We proceed by reducito ad absurdam: Suppose there were no $N(I)$ with the property stated in the Proposition. Then there would be an increasing sequence of positive integers $\left\{n_{i}\right\}$ with $n_{i} \uparrow \infty$ and a sequence of pairs of points in the parameter space 
$\left\{\left(\varepsilon_{i}^{0}, \varepsilon_{i}^{1}\right)\right\}$ such that $\lambda_{k}\left(\varepsilon_{i}^{0}\right)=\lambda_{k}\left(\varepsilon_{i}^{1}\right)$ for all $k=1, \ldots, n_{i}$, but $\lambda_{n_{k}+1}\left(\varepsilon_{i}^{0}\right) \neq$ $\lambda_{n_{k}+1}\left(\varepsilon_{i}^{1}\right)$. Since $K$ (and therefore $K \times K$ ) is compact, there is a subsequence of $\left\{\left(\varepsilon_{i}^{0}, \varepsilon_{i}^{1}\right)\right\}$ (which we continue to denote $\left\{\left(\varepsilon_{i}^{0}, \varepsilon_{i}^{1}\right)\right\}$ ) which converges, say to $\left(\varepsilon_{*}^{0}, \varepsilon_{*}^{1}\right) \in K \times K$. By continuity of the symmetric functions of the eigenvalues in a neighborhood of $\left(\varepsilon_{*}^{0}, \varepsilon_{*}^{1}\right)$ as explained above, we must have $\operatorname{Spec}\left(\varepsilon_{*}^{0}\right)=$ $\operatorname{Spec}\left(\varepsilon_{*}^{1}\right)$. We choose the $\left\{a_{i}\right\}$ of the previous paragraph to be appropriate for $\operatorname{Spec}\left(\varepsilon_{*}^{0}\right)$ and $\left.\operatorname{Spec}\left(\varepsilon_{*}^{1}\right)\right)$, and assume that $n_{i}=\#\left\{\lambda \in \operatorname{Spec}\left(\varepsilon_{*}^{0}\right) \mid \lambda<a_{i}\right\}$, without loss of generality.

Let $\mathscr{O}$ be the ring of germs at $\left(\varepsilon_{*}^{0}, \varepsilon_{*}^{1}\right)$ of real analytic functions on $K \times K$. We consider the increasing sequence of ideals $\left\{\mathscr{I}_{m}\right\}$ in $\mathscr{O}$, where $\mathscr{I}_{m}$ is generated by the set $\left\{\sigma_{i, j}\left(\varepsilon_{0}\right)-\sigma_{i j}\left(\varepsilon_{1}\right) \mid i \leq m\right\}$, where $\sigma_{i, j}(\varepsilon)$ is the $j$ th symmetric function of the eigenvalues of the Laplacian of $g(\varepsilon)$ which lie between $a_{i}$ and $a_{i+1}$ (for $j$ between 1 and $n_{i+1}-n_{i}$ ). Since $\mathscr{I}_{m}$ is generated by finitely many functions, there is a neighborhood $U_{m}$ of $\left(\varepsilon_{*}^{0}, \varepsilon_{*}^{1}\right)$ on which all of the series of these analytic functions converge-the common zeroes of these functions comprise an analytic set $A_{m} \subset U_{m}$. Now we can use the fact that $\mathscr{O}$ is a Noetherian ring (see [N], Chapter 2) to conclude that there is an integer $M$ such that $\mathscr{I}_{m}=\mathscr{I}_{M}$ for all $m \geq M$; in other words, $A_{m}=A_{M} \cap U_{m}$ for $m \geq M$. Note that $\operatorname{dim} A_{M} \geq 1$ since $A_{M}$ contains infinitely many distinct points (those of the sequence). $\mathscr{O}$ being Noetherian also implies that $A_{M}$ has only finitely many irreducible components (i.e., connected components, see [N], Chapter 3). We may thus assume (by passing to a subsequence again) that all the points in our sequence and the limit lie in the same connected component of $A_{M}$. Now choose $m>M$, then there is an $i$ so that $\left(\varepsilon_{i}^{0}, \varepsilon_{i}^{1}\right) \in A_{m}$, therefore there is an analytic curve, completely contained in $A_{m}$, which connects $\left(\varepsilon_{i}^{0}, \varepsilon_{i}^{1}\right)$ to $\left(\varepsilon_{*}^{0}, \varepsilon_{*}^{1}\right)$. But Theorem 1 of Chapter 2 of $[R]$ tells us that, as a function of a single parameter, branches of each eigenvalue may be chosen to be analytic. For $\left(\varepsilon^{0}, \varepsilon^{1}\right)$ on the curve and close enough to $\left(\varepsilon_{*}^{0}, \varepsilon_{*}^{1}\right)$, any given eigenvalue of $g\left(\varepsilon^{0}\right)$ is equal to its counterpart for $g\left(\varepsilon^{1}\right)$, thus, the analytic function which is the difference of these eigenvalues is identically zero on an open part of our curve near $\left(\varepsilon_{*}^{0}, \varepsilon_{*}^{1}\right)$. This would imply that all the metrics on our curve would be isospectral, which contradicts our choice of $\left\{\left(\varepsilon_{i}^{0}, \varepsilon_{i}^{1}\right)\right\}$.

It is interesting to note how the number $N$ of eigenvalues required in the conclusion of the Proposition can vary as we consider different (even closely related) families. For example, the spectrum of a rectangle in the Euclidean plane with length $L$ and height $H$ is given by the set of numbers

$$
\left\{\frac{p^{2} \pi^{2}}{L^{2}}+\frac{q^{2} \pi^{2}}{H^{2}}, p, q=1,2, \ldots\right\} .
$$

Thus, from the first two eigenvalues, we can determine $L$ and $H$, and so for any family of rectangles, $N=2$. On the other hand, if we consider rectangular 
flat tori, then the eigenvalues are

$$
\left\{\frac{p^{2} \pi^{2}}{L^{2}}+\frac{q^{2} \pi^{2}}{H^{2}}, p, q=0,1,2, \ldots\right\} .
$$

If $H$ is very small compared to $L$, say $H=L / M$, then the first $M$ eigenvalues of the torus all come from choosing $q=0$, so we cannot determine $H$ until we have "heard" at least $M+1$ eigenvalues. As $M \rightarrow \infty$ then, so does $N$. This seems to be an indication of the general picture, in that for convex bounded domains in Euclidean space, arguments similar to those given in the next section enable us to use the first two eigenvalues to find a compact set in which the domain is contained. But for manifolds without boundary, there can be no such results without more geometric assumptions.

\section{TRIANGLES, ETC.}

We specialize the result of the previous section first to triangles in the Euclidean plane. Our motivation for doing this comes from recent attention and progress on the problem of identifying triangles spectrally (see [C] and [D]). The work done up to now has made use of asymptotics, and thus information about infinitely many eigenvalues-we show that finitely many should suffice.

For triangles the above proposition is applicable, since we can consider any Euclidean triangle $T$ to have as underlying manifold the "standard triangle" with vertices $(0,0),(0,1)$, and $(1,0)$, and metric given by the pullback of the standard Euclidean metric by the obvious linear map from the "standard triangle" to $T$. It is then clear that the metric is an analytic function of the lengths of two sides of the triangle and the angle between them, for example.

Theorem 1. Let $T_{0}$ be a (Euclidean) triangle. There is an integer $N$, which depends only on the first two eigenvalues of $T_{0}$ such that if $T_{1}$ is another triangle with $\lambda_{1}\left(T_{0}\right)=\lambda_{1}\left(T_{1}\right), \ldots, \lambda_{N}\left(T_{0}\right)=\lambda_{N}\left(T_{1}\right)$, then $T_{0}$ and $T_{1}$ are isospectral.

Proof. We need only show that the set of triangles with a given $\lambda_{1}$ and $\lambda_{2}$ is compact. Clearly, this will be accomplished if we can find an upper bound for the length of the longest side and a lower bound for the length of the shortest side of such a triangle. To obtain these bounds, first recall that the eigenvalues of a domain are "monotonic" functions of the domain, i.e., if $D_{1} \subset D_{2}$, then $\lambda_{i}\left(D_{1}\right) \geq \lambda_{i}\left(D_{2}\right)$ for all $i>0$. Now, observe that we can inscribe the triangle in a rectangle of length $L$ and height $H$, where $L$ is the length of the longest side of the triangle and $H$ is the height of the triangle off the longest side. Recall that the first eigenvalue of this rectangle is $\pi^{2}\left(H^{-2}+L^{-2}\right)$. Since the first eigenvalue of the rectangle is smaller than that of the triangle, we have

$$
\lambda_{1}(T) \geq \pi^{2} / H^{2},
$$

which gives a lower bound for the length of the triangle's shortest side. Now, inscribe a rectangle of height $\alpha H$ and length $(1-\alpha) L$ inside the triangle (the length of the rectangle is along the longest side of the triangle). Since $\lambda_{2}(T)$ 
is strictly bigger than $\lambda_{1}(T)$, we can find an $\alpha$ less than 1 so that $\alpha^{2} \lambda_{2}(T)>$ $\lambda_{1}(T)$. The fact that the second eigenvalue of the inscribed rectangle (which is the smaller of $\pi^{2}\left\{4(\alpha H)^{-2}+((1-\alpha) L)^{-2}\right\}$ and $\left.\pi^{2}\left\{(\alpha H)^{-2}+4((1-\alpha) L)^{-2}\right\}\right)$ is bigger than the second eigenvalue of the triangle, combined with the estimate above for $H$ yields

$$
\lambda_{2}(T)-\frac{1}{\alpha^{2}} \lambda_{1}(T) \leq \frac{4 \pi^{2}}{(1-\alpha)^{2} L^{2}} .
$$

This gives the required upper bound for $L$.

Theorem 2. Let $E_{0}$ be the interior of an ellipsoid in $\mathbb{R}^{n}$. There is an integer $N$, which depends only on the first two eigenvalues of $E_{0}$ such that if $E_{1}$ is the interior of another ellipsoid with $\lambda_{1}\left(E_{0}\right)=\lambda_{1}\left(E_{1}\right), \ldots, \lambda_{N}\left(E_{0}\right)=\lambda_{N}\left(E_{1}\right)$, then $E_{0}$ and $E_{1}$ are isospectral.

Proof. As for triangles, we can consider an ellipsoid to have as underlying manifold the unit ball in $\mathbb{R}^{n}$, with the metric obtained by pulling the Euclidean metric back via the obvious (diagonal) linear map. Thus, the metrics of ellipsoids vary analytically as functions of the lengths of the principal axes. We need to show that ellipsoids with given $\lambda_{1}$ and $\lambda_{2}$ comprise a compact set. As with triangles, this means finding a lower bound for the length $a_{1}$ of the shortest principal axis, and an upper bound for the length $a_{n}$ of the longest. The first task is easy, since the ellipsoid can be inscribed within a rectangular box whose shortest side has the same length as the shortest axis. Thus,

$$
\lambda_{1}(E) \geq \pi^{2} / a_{1}^{2}
$$

as before. To proceed further, we need a slightly better estimate of $\lambda_{1}$. The ellipsoid can also be inscribed in the right cylinder of length $a_{n}$ whose cross section is the $(n-1)$-dimensional ellipsoid with axes $a_{1}, \ldots, a_{n-1}$. If $\mu_{1}$ is the first eigenvalue of the interior of this $(n-1)$-dimensional ellipsoid, then we have $\lambda_{1}(E) \geq \mu_{1}$. As with the triangles, we will now inscribe a right cylinder whose cross section is the $n-1$-dimensional ellipsoid with axes $\alpha a_{1}, \ldots, \alpha a_{n-1}$ for $\alpha<1$ such that $\lambda_{1}(E)<\alpha^{2} \lambda_{2}(E)$. Note that the first eigenvalue of this $(n-1)-$ dimensional ellipsoid is $\mu_{1} / \alpha^{2}$. The length of the cylinder is $\left(1-\alpha^{2}\right)^{1 / 2} a_{n}$, so as above we conclude

$$
\lambda_{2}(E)-\frac{1}{\alpha^{2}} \lambda_{1}(E) \leq \frac{4 \pi^{2}}{\left(1-\alpha^{2}\right) a_{n}^{2}} .
$$

This completes the proof.

This line of proof can be easily generalized to other finite-dimensional families of subsets of Euclidean space (e.g., simplices, parallelopipeds, etc).

\section{REFERENCES}

[C] P. Chang, Ph.D. Thesis, University of Pennsylvania, 1988.

[D] C. Durso, Ph.D. Thesis, MIT, 1988. 
[GS] P. Garabedian and M. Schiffer, Convexity of domain functionals, J. Analyse Math. 2 (1952-3), 283-358.

[K] M. Kac, Can one hear the shape of a drum?, Amer. Math. Monthly, 73 (1966), 1-23.

[Ko] T. Kato, Perturbation theory for linear operators, Springer-Verlag, Berlin, 1980.

[N] R. Narasimhan, Introduction to the theory of analytic spaces, Springer-Lecture Notes 25 (1966).

[P] M. Protter, Can one hear the shape of a drum? Revisited, SIAM Rev. 29 (1987), 185-197.

[R] F. Rellich, Perturbation theory of eigenvalue problems, Gordon and Breach, New York, 1969.

Department of Mathematics, University of Pennsylvania, Philadelphia, PenNSYlvania 19104 